\title{
A COMPLETE SOLUTION OF THE CONVERGENCE PROBLEM FOR CONTINUED FRACTIONS
}

RALPH E. LANE

1. Introduction. In this paper we consider continued fractions of the form

$$
f_{1}+\frac{a_{1}}{b_{1}-\frac{a_{2}}{b_{2}-\frac{a_{3}}{b_{3}-\cdots}}},
$$

where $f_{1}$ is a number and (for $\left.p=1,2,3, \cdots\right) a_{p}$ is a nonzero number ${ }^{1}$ and $b_{p}$ is a number.

If $f=\left\{f_{p}\right\}_{p=1}^{\infty}$ is the sequence of approximants of (1.1), then for $f$ to converge, it is necessary that there exist a positive integer $n$, a nonzero number $a_{n}^{\prime}$, and a number $b_{n}^{\prime}$ such that the sequence of approximants of the continued fraction

$$
f_{n}+\frac{a_{n}^{\prime}}{b_{n}^{\prime}-\frac{a_{n+1}}{b_{n+1}-\frac{a_{n+2}}{b_{n+2}-\cdots}}}
$$

is $\left\{f_{n+p-1}\right\}_{p=1}^{\infty}$, where $f_{n+p-1} \neq \infty$ for $p=1,2,3, \cdots$. Consequently the following theorem is a complete solution of the convergence problem for continued fractions.

TheOREM 1. For a continued fraction $F$ to have only finite approximants and to converge, it is necessary and sufficient that there exist a continued fraction (1.1) equivalent to $F$ and a sequence $s$ of numbers such that

(i) $0<s_{p}<1$ for $p=1,2,3, \cdots$, and

(ii) $s_{1}\left|b_{1}\right|>\left|b_{1}-1\right|$, and

(iii) if, for each positive integer $p$,

$$
e_{p}=\left[\frac{s_{p+1}}{1-s_{p+1}^{2}}-\left|a_{p+1}-b_{p+1}+\frac{1}{1-s_{p+1}^{2}}\right|\right]\left[\frac{1}{s_{p}\left|a_{p+1}\right|}\right],
$$

Received by the editors February 11, 1952.

${ }^{1}$ For a solution of the convergence problem in the case in which one (or more) of the partial numerators $a_{p}$ is zero, see H.S. Wall, Analytic theory of continued fractions, New York, 1948, p. 26. 
then $e_{p} \geqq 1$ for $p=1,2,3, \cdots$, and $\sum_{p=1}^{\infty}\left(e_{p}-1\right)$ diverges.

It is interesting that the concepts which we use in the proof of this theorem are essentially the ones which were used in carrying out Wall's program (loc. cit., p. 5) for positive definite continued fractions; namely, a sequence of linear fractional transformations which generates the continued fraction, and a "nest of circles" which provides a sequence of bounds for the approximants of the continued fraction.

2. Lemmas. We use the following notation:

(i) The generator of (1.1) is the sequence, $t$, of linear fractional transformations such that $t_{1}(u)=f_{1}+a_{1} /\left(b_{1}-u\right)$ and $t_{p}^{-1} t_{p+1}(u)$ $=a_{p+1} /\left(b_{p+1}-u\right), p=1,2,3, \cdots$.

(ii) The sequence of approximants of (1.1) is the sequence $f$ $=t(\infty)$; i.e., $f_{p}=t_{p}(\infty)$ for $p=1,2,3, \cdots$. If $f_{p} \neq \infty$, then we write $t_{p}(u)=f_{p}+g_{p} /\left(h_{p}-u\right)$.

(iii) If $f$ is bounded, then $B_{f}$ is the set such that $R \in B_{f}$ if and only if $R$ is a sequence $\left\{R_{p}\right\}_{p=1}^{\infty}$ such that if $p$ is a positive integer, then $R_{p}$ is a circle plus its interior, and $R_{p} \supset R_{p+1}$, and $f_{p}$ is an interior point of $R_{p}$.

Lemma 1. If $R \in B_{f}$, then there exist a sequence $s$ of positive numbers less than 1 and a sequence $q$ of nonzero numbers such that if $p$ is a positive integer, then $t_{p}^{-1}\left(R_{p}\right)$ is the region (a circle plus its exterior) defined by the inequality

$$
s_{p}|u| \leqq\left|u-q_{p}\right|
$$

which is equivalent to the inequality

$$
\left|u-\frac{q_{p}}{1-s_{p}^{2}}\right| \geqq \frac{s_{p}\left|q_{p}\right|}{1-s_{p}^{2}} .
$$

Proof. Suppose that $R \in B_{f}$. By hypothesis, $f_{p}=t_{p}(\infty)$ is an interior point of $R_{p}$, so that $\infty$ is an interior point of $t_{p}^{-1}\left(R_{p}\right)$; hence $t_{p}^{-1}\left(R_{p}\right)$ is a circle plus its exterior. Moreover, $f_{p+1}=t_{p}(0)$ is an interior point of $R_{p+1}$ and a fortiori of $R_{p}$; hence 0 is an interior point of $t_{p}^{-1}\left(R_{p}\right)$. Let $q_{p}$ denote the inversion of the origin in the boundary of $t_{p}^{-1}\left(R_{p}\right)$. Then there exists a number $s_{p}$ such that $0<s_{p}<1$ and such that $t_{p}^{-1}\left(R_{p}\right)$ is the region defined by $s_{p}|u| \leqq\left|u-q_{p}\right|$. If both sides of this inequality are squared, the result can be written as $\left(1-s_{p}^{2}\right) u \bar{u}$ $-\bar{q}_{p} u-q_{p} \bar{u}+q_{p} \bar{q}_{p} \geqq 0$, which is equivalent to the inequality $\left|u-q_{p} /\left(1-s_{p}^{2}\right)\right| \geqq s_{p}\left|q_{p}\right| /\left(1-s_{p}^{2}\right)$. This completes the proof. 
LEMмA 2. For the sequence of approximants of (1.1) to be bounded, it is necessary and sufficient that there exist a sequence $s$ of numbers and a sequence $q$ of numbers such that

(i) $0<s_{p}<1$ and $q_{p} \neq 0, p=1,2,3, \cdots$, and

(ii) $s_{1}\left|b_{1}\right|>\left|b_{1}-q_{1}\right|$, and

(iii) $\frac{s_{p}\left|a_{p+1}\right|}{\left|q_{p} q_{p+1}\right|}+\left|\frac{a_{p+1}}{q_{p} q_{p+1}}-\frac{b_{p+1}}{q_{p+1}}+\frac{1}{1-s_{p+1}^{2}}\right| \leqq \frac{s_{p+1}}{1-s_{p+1}^{2}}$

for $p=1,2,3, \cdots$.

Proof. Suppose that $f$, the sequence of approximants of (1.1), is bounded. Let $R$ denote a sequence in $B_{f}$. By Lemma 1 , there exist sequences $s$ and $q$ such that (i) holds and such that $t_{p}^{-1}\left(R_{p}\right)$ is the region defined by $s_{p}|u| \leqq\left|u-q_{p}\right|, p=1,2,3, \cdots$. Since $t_{1}\left(b_{1}\right)=\infty$, condition (ii) is merely the condition for $R_{1}$ to be a circle plus its interior. Now $t_{p+1}^{-1}\left(R_{p}\right)$ is defined by the inequality $s_{p}\left|a_{p+1} /\left(b_{p+1}-u\right)\right|$ $\leqq\left|a_{p+1} /\left(b_{p+1}-u\right)-q_{p}\right|$, which is equivalent to the inequality $\left|u-\left(b_{p+1}-a_{p+1} / q_{p}\right)\right| \geqq s_{p}\left|a_{p+1} / q_{p}\right|$. Hence (iii) is the condition that

$$
t_{p+1}^{-1}\left(R_{p}\right) \supset t_{p+1}^{-1}\left(R_{p+1}\right) \text {, or } R_{p} \supset R_{p+1},
$$

for $p=1,2,3, \cdots$.

Suppose, on the other hand, that there exist such sequences $s$ and $q$. For each positive integer $p$; let $t_{p}^{-1}\left(R_{p}\right)$ denote the region defined by the inequality $s_{p}|u| \leqq\left|u-q_{p}\right|$. Then $R_{1}$ is a circle plus its interior; and if $p$ is a positive integer, then $R_{p} \supset R_{p+1}$ and $f_{p}=t_{p}(\infty)$ is in $R_{p}$, so that each member of $f$ is in $R_{1}$, and consequently $f$ is bounded. This completes the proof.

We now introduce the following notation in addition to that established at the beginning of this section. If $R \in B_{f}$, and if $p$ is a positive integer, then

(iv) $c_{p}$ is the center and $r_{p}$ the radius of $R_{p}$; i.e., $R_{p}$ is defined by the inequality $\left|u-c_{p}\right| \leqq r_{p}$; and

(v) if $q=p$ or if $q=p+1$, then $c_{q, p+1}$ is the center and $r_{q, p+1}$ the radius of $t_{p+1}^{-1}\left(R_{q}\right)$; i.e., $t_{p+1}^{-1}\left(R_{q}\right)$ is defined by the inequality $\left|u-c_{q, p+1}\right|$ $\geqq r_{q, p+1}$. We observe that $\left|c_{p, p+1}-c_{p+1, p+1}\right| \leqq r_{p+1, p+1}-r_{p, p+1}$.

LEMMA 3. In order that $r_{p} \rightarrow 0$ as $p \rightarrow \infty$, it is necessary and sufficient that $\sum_{p=1}^{\infty}\left(1-r_{p+1} / r_{p}\right)$ diverge, and it is necessary and sufficient that $\sum_{p=1}^{\infty}\left(r_{p} / r_{p+1}-1\right)$ diverge.

Proof. By hypothesis, $R_{p} \supset R_{p+1}$, so that $\left\{r_{p}\right\}_{p=1}^{\infty}$ is a nonincreasing sequence of positive numbers. Let $r$ denote the number such that $r_{p} \rightarrow r$ as $p \rightarrow \infty$. We write 


$$
\begin{aligned}
\frac{r_{p+1}}{r_{1}} & =\frac{r_{2}}{r_{1}} \cdot \frac{r_{3}}{r_{2}} \ldots \cdot \frac{r_{p+1}}{r_{p}} \\
& =\left(1-\frac{r_{1}-r_{2}}{r_{1}}\right)\left(1-\frac{r_{2}-r_{3}}{r_{2}}\right) \cdots\left(1-\frac{r_{p}-r_{p+1}}{r_{p}}\right) .
\end{aligned}
$$

It follows that $r=0$ if and only if $\sum_{p=1}^{\infty}\left(1-r_{p+1} / r_{p}\right)$ diverges. From a similar argument about the ratios $r_{1} / r_{p+1}$ it follows that $r=0$ if and only if $\sum_{p=1}^{\infty}\left(r_{p} / r_{p+1}-1\right)$ diverges. This completes the proof.

LemMa 4. If $p$ is a positive integer, and if $q=p$ or $q=p+1$, then $c_{q, p+1}=h_{p+1}-g_{p+1}\left(\bar{f}_{p+1}-\bar{c}_{q}\right) /\left(r_{q}^{2}-\left|f_{p+1}-c_{q}\right|^{2}\right)$ and

$$
r_{q, p+1}=\left|g_{p+1}\right| r_{q} /\left(r_{q}^{2}-\left|f_{p+1}-c_{q}\right|^{2}\right) \text {. }
$$

Proof. By hypothesis, $R_{q}$ is defined by the inequality $\left|u-c_{q}\right| \leqq r_{q}$, whence $t_{p+1}^{-1}\left(R_{q}\right)$ is defined by the inequality $\left|t_{p+1}(u)-c_{q}\right| \leqq r_{q}$, which is equivalent to the inequality $\left|\left(f_{p+1}-c_{q}\right)\left(u-h_{p+1}\right)-g_{p+1}\right|$ $\leqq r_{q}\left|u-h_{p+1}\right|$. If $f_{p+1}=c_{q}$, then $c_{q, p+1}=h_{p+1}$ and $r_{q, p+1}=\left|g_{p+1}\right| / r_{q}$, and the lemma holds. Suppose, however, that $f_{p+1} \neq c_{q}$. Then $t_{p+1}^{-1}\left(R_{q}\right)$ is defined by the inequality $\left|u-h_{p+1}-g_{p+1} /\left(f_{p+1}-c_{q}\right)\right|$ $\leqq\left|u-h_{p+1}\right| r_{q} /\left|f_{p+1}-c_{q}\right|$, which is equivalent, since $\left|f_{p+1}-c_{q}\right|<r_{q}$, to the inequality

$$
\begin{aligned}
\mid u-\left[h_{p+1}-g_{p+1}\left(\bar{f}_{p+1}-\bar{c}_{q}\right) /\left(r_{q}^{2}-\mid\right.\right. & \left.\left.f_{p+1}-\left.c_{q}\right|^{2}\right)\right] \mid \\
& \geqq\left|g_{p+1}\right| r_{q} /\left(r_{q}^{2}-\left|f_{p+1}-c_{q}\right|^{2}\right),
\end{aligned}
$$

so that the lemma follows from the definitions of $c_{q, p+1}$ and $r_{q, p+1}$. This completes the proof.

Lemma 5. If $p$ is a positive integer, then

$$
\frac{r_{p+1, p+1}}{r_{p, p+1}} \geqq \frac{r_{p}-\left|c_{p}-c_{p+1}\right|}{r_{p+1}} \geqq 1,
$$

and

$$
\frac{r_{p}}{r_{p+1}} \geqq \frac{r_{p+1, p+1}-\left|c_{p, p+1}-c_{p+1, p+1}\right|}{r_{p, p+1}} \geqq 1 .
$$

Proof. We shall prove statement (i): statement(ii) can be proved by the same kind of argument. By hypothesis, $R_{p} \supset R_{p+1}$, whence $r_{p}-r_{p+1} \geqq\left|c_{p}-c_{p+1}\right|$, and $\left(r_{p}-\left|c_{p}-c_{p+1}\right|\right) / r_{p+1} \geqq 1 ;$ moreover, $t_{p+1}^{-1}\left(R_{p}\right) \supset t_{p+1}^{-1}\left(R_{p+1}\right)$, and consequently $r_{p+1, p+1} \geqq r_{p, p+1}$. If $r_{p+1}=r_{p}$, then $R_{p}=R_{p+1}$ and $t_{p+1}^{-1}\left(R_{p}\right)=t_{p+1}^{-1}\left(R_{p+1}\right)$, so that (i) holds with actual equality throughout. Suppose, however, that $r_{p+1}<r_{p}$. By hypothesis, 
$\left|f_{p+1}-c_{p}\right|<r_{p}$ and $\left|f_{p+1}-c_{p+1}\right|<r_{p+1} ;$ and from Lemma 4 it follows that

$$
\frac{r_{p+1, p+1}}{r_{p, p+1}}=\frac{r_{p+1}}{r_{p}} \cdot \frac{r_{p}^{2}-\left|f_{p+1}-c_{p}\right|^{2}}{r_{p+1}^{2}-\left|f_{p+1}-c_{p+1}\right|^{2}} .
$$

Let $k$ denote the number such that $k r_{p+1} / r_{p}=\left(r_{p}-\left|c_{p}-c_{p+1}\right|\right) / r_{p+1}$; then $k r_{p+1} / r_{p} \geqq 1$, and $k \geqq r_{p} / r_{p+1}>1$. Let $K$ denote the common part of the region defined by the inequality

$$
\frac{r_{p}^{2}-\left|u-c_{p}\right|^{2}}{r_{p+1}^{2}-\left|u-c_{p+1}\right|^{2}} \geqq k
$$

and the interior of $R_{p+1}$; then $c_{p+1} \in K$, and it can readily be seen that $K$ is actually a region. For $\left|u-c_{p+1}\right|<r_{p+1}$, the inequality (2.2) is equivalent to the inequality

$$
\left|u-\frac{c_{p}-k c_{p+1}}{1-k}\right|^{2} \geqq \frac{(k-1)\left(k r_{p+1}^{2}-r_{p}^{2}\right)+k\left|c_{p}-c_{p+1}\right|^{2}}{(k-1)^{2}} .
$$

But $k r_{p+1}^{2}-r_{p}^{2}=r_{p}\left(r_{p}-\left|c_{p}-c_{p+1}\right|\right)-r_{p}^{2}=-r_{p}\left|c_{p}-c_{p+1}\right|$, so that

$$
\begin{aligned}
(k-1)\left(k r_{p+1}^{2}-r_{p}^{2}\right) & +k\left|c_{p}-c_{p+1}\right|^{2} \\
& =k\left|c_{p}-c_{p+1}\right|^{2}-r_{p}\left|c_{p}-c_{p+1}\right|(k-1) \\
& =r_{p}\left|c_{p}-c_{p+1}\right|-k\left(r_{p}-\left|c_{p}-c_{p+1}\right|\right)\left|c_{p}-c_{p+1}\right| \\
& =\left|c_{p}-c_{p+1}\right|\left[r_{p}-r_{p}\left(r_{p}-\left|c_{p}-c_{p+1}\right|\right)^{2} / r_{p+1}^{2}\right] \\
& =r_{p}\left|c_{p}-c_{p+1}\right|\left[r_{p+1}^{2}-\left(r_{p}-\left|c_{p}-c_{p+1}\right|\right)^{2}\right] / r_{p+1}^{2} \\
& \leqq 0
\end{aligned}
$$

since $r_{p+1} \leqq r_{p}-\left|c_{p}-c_{p+1}\right|$. Hence $K$ is merely the interior of $R_{p+1}$. Since $f_{p+1}$ is an interior point of $R_{p+1}$, it follows from (2.1) that

$$
\frac{r_{p+1, p+1}}{r_{p, p+1}} \geqq \frac{r_{p+1}}{r_{p}} k=\frac{r_{p}-\left|c_{p}-c_{p+1}\right|}{r_{p+1}} .
$$

This completes the proof.

LEMMA 6. If $c_{p}=c_{p+1}$, and if $r_{p+1} \geqq 3\left|f_{p+1}-c_{p}\right|$, then

$$
\frac{r_{p+1, p+1}-\left|c_{p, p+1}-c_{p+1, p+1}\right|}{r_{p, p+1}}-1 \geqq \frac{1}{3}\left(\frac{r_{p}}{r_{p+1}}-1\right) .
$$

Proof. By Lemma 4 and the hypothesis that $c_{p}=c_{p+1}$, 


$$
\begin{aligned}
\frac{\left|c_{p, p+1}-c_{p+1, p+1}\right|}{r_{p, p+1}} & =\frac{\left|f_{p+1}-c_{p}\right|}{r_{p, p+1}}\left|\frac{r_{p+1, p+1}}{r_{p+1}}-\frac{r_{p, p+1}}{r_{p}}\right| \\
& =\frac{\left|f_{p+1}-c_{p}\right|}{r_{p+1}}\left|\frac{r_{p+1, p+1}}{r_{p, p+1}}-\frac{r_{p+1}}{r_{p}}\right| .
\end{aligned}
$$

But $r_{p+1, p+1} / r_{p, p+1} \geqq 1 \geqq r_{p+1} / r_{p}$. Hence

$$
\frac{\left|c_{p, p+1}-c_{p+1, p+1}\right|}{r_{p, p+1}}=\frac{\left|f_{p+1}-c_{p}\right|}{r_{p+1}}\left(\frac{r_{p+1, p+1}}{r_{p, p+1}}-\frac{r_{p+1}}{r_{p}}\right)
$$

consequently, if

$$
e_{p}=\frac{r_{p+1, p+1}-\left|c_{p, p+1}-c_{p+1, p+1}\right|}{r_{p, p+1}}
$$

then

$$
\begin{aligned}
e_{p}-1= & \left(\frac{r_{p+1, p+1}}{r_{p, p+1}}-1\right) \\
& -\frac{\left|f_{p+1}-c_{p}\right|}{r_{p+1}}\left[\left(\frac{r_{p+1, p+1}}{r_{p, p+1}}-1\right)+\left(1-\frac{r_{p+1}}{r_{p}}\right)\right] \\
\geqq & \frac{2}{3}\left(\frac{r_{p+1, p+1}}{r_{p, p+1}}-1\right)-\frac{1}{3}\left(1-\frac{r_{p+1}}{r_{p}}\right) .
\end{aligned}
$$

Now $r_{p} / r_{p+1}-1 \geqq 1-r_{p+1} / r_{p} \geqq 0$; moreover, from (i) of Lemma 5 and from the hypothesis that $c_{p}=c_{p+1}$, it follows that $r_{p+1, p+1} / r_{p, p+1}$ $\geqq r_{p} / r_{p+1}$. Hence $e_{p}-1 \geqq\left(r_{p} / r_{p+1}-1\right) / 3$. This completes the proof.

3. Proof of the theorem. Let $F$ denote a continued fraction

$$
f_{1}+\frac{a_{1}^{\prime}}{b_{1}^{\prime}-\frac{a_{2}^{\prime}}{b_{2}^{\prime}-\cdots}}
$$

where $a_{p}^{\prime} \neq 0, p=1,2,3, \cdots$. Let $f$ denote the sequence of approximants of $F$.

Suppose first that there exist a continued fraction (1.1), which is equivalent to $F$, and a sequence $s$ such that conditions (i), (ii), and (iii) of the theorem hold. It follows from Lemma 2 (with $q_{p}=1$ for $p=1,2,3, \cdots)$ that the sequence of approximants of $F$ is bounded. For each positive integer $p$, let $t_{p}^{-1}\left(R_{p}\right)$ be the region defined by the inequality $s_{p}|u| \leqq|u-1|$. By Lemma $1, c_{p+1, p+1}=1 /\left(1-s_{p+1}^{2}\right)$ and $r_{p+1, p+1}=s_{p+1} /\left(1-s_{p+1}^{2}\right)$; and from the proof of Lemma $2, c_{p, p+1}$ 
$=b_{p+1}-a_{p+1}$ and $r_{p, p+1}=s_{p}\left|a_{p+1}\right|$. Hence

$$
e_{p}=\frac{r_{p+1, p+1}-\left|c_{p, p+1}-c_{p+1, p+1}\right|}{r_{p, p+1}} .
$$

By hypothesis, $\sum_{p=1}^{\infty}\left(e_{p}-1\right)$ diverges; by Lemma $5, \sum_{p=1}^{\infty}\left(r_{p} / r_{p+1}-1\right)$ diverges; and by Lemma 3, $r_{p} \rightarrow 0$ as $p \rightarrow \infty$. Hence the common part of the regions $R_{p}$ is a point, $c$, and $f_{p} \rightarrow c$ as $p \rightarrow \infty$; that is to say, $F$ converges.

Suppose that $f$ is bounded and that $F$ converges. Let $c$ denote the number such that $f_{p} \rightarrow c$ as $p \rightarrow \infty$. For each positive integer $p$, let $c_{p}=c$, let $r_{p}=3$ l.u.b. $q \geqq p\left|f_{q}-c\right|$, and let $R_{p}$ denote the region defined by $|u-c| \leqq r_{p}$. Then $R \in B_{f}$. Let $\tau_{1}(u)=f_{1}+a_{1}^{\prime} /\left(b_{1}^{\prime}-u\right)$ and $\tau_{p}^{-1} \tau_{p+1}(u)=a_{p+1}^{\prime} /\left(b_{p+1}^{\prime}-u\right)$ for $p=1,2,3, \cdots$, and let $s$ and $q$ denote sequences such that (cf. Lemma 1) $\tau_{p}^{-1}\left(R_{p}\right)$ is defined by $s_{p}|u|$ $\leqq\left|u-q_{p}\right|$. Let $a_{1}=a_{1}^{\prime} / q_{1}$, and for each positive integer $p$, let $b_{p}$ $=b_{p}^{\prime} / q_{p}$ and $a_{p+1}=a_{p+1}^{\prime} / q_{p} q_{p+1}$. We have now found a continued fraction (1.1) which is equivalent to $F$ and a sequence $s$ such that (cf. Lemma 2) the conditions (i) and (ii) of the theorem hold and $e_{p} \geqq 1$ for $p=1,2,3, \cdots$. Moreover, $t_{p}^{-1}\left(R_{p}\right)$ is defined by the inequality $s_{p}|u| \leqq|u-1|$, so that (3.1) holds. By hypothesis and by construction, $r_{p} \rightarrow 0$ as $p \rightarrow \infty$, so that (Lemma 3) $\sum_{p=1}^{\infty}\left(r_{p} / r_{p+1}-1\right)$ diverges. From (3.1) and Lemma 6 , it follows that $\sum_{p=1}^{\infty}\left(e_{p-1}\right)$ diverges. This completes the proof.

The University of Texas 